\title{
What is universal and what differs in language development?
}

\author{
Gillian S. Forrester ${ }^{1} \&$ Michael S. C. Thomas ${ }^{2}$ \\ ${ }^{1}$ Department of Psychology, University of Westminster, London, UK \\ ${ }^{2}$ Developmental Neurocognition Lab, Birkbeck, University of London, UK
}

Running head: Species universals and individual differences

Corresponding author:

Dr. Gillian S. Forrester

Department of Psychology

University of Westminster

115 New Cavendish Street

London W1W 6UW

United Kingdom

g.forrester@westminster.ac.uk 
Goldin-Meadow (2015) presents an exceptional synthesis of work from studies of children acquiring language under variable circumstances of input or processing abilities. Deaf children who acquire homesign without any wellformed model from which to learn language represent a powerful example. Goldin-Meadow argues that the resilient properties of language that nevertheless emerge include simple syntactic structures, hierarchical organisation, markers modulating the meaning of sentences, and socialcommunicative functions. Among the fragile or input-dependent properties are the orders that the language follows, the parts into which words are decomposed, and the features that distinguish nominals from predicates. Separation of these two types of properties poses questions concerning the innate constraints on language acquisition (perhaps these equate to the resilient properties) and concerning the specificity of processes to language (e.g., whether properties such as hierarchical organisation are specific to language or originate in the structure of thought).

The study of the resilient properties of human language in the face of adversity, and the relation of these properties to the information that is encoded in the human genome, represent a research strategy that draws inferences about species universals (properties that all humans share) from data about individual differences (factors that make humans different from one another). In the following, we suggest three reasons to be cautious about this approach.

\section{Comparisons of typical and atypical development may not reveal the 'hidden' properties of typical development}


The first concerns the relation of atypical systems to typical systems. The method adopted assumes that what is revealed in a case of language acquisition without a language model is the resilient properties that are otherwise hidden in the presence of a language model: typical development represents the addition of resilient properties and the fragile properties filled in by the language model. Remove the latter in the atypical case, and the former is revealed. This is to employ what is sometimes called the 'transparency' assumption. In the developmental case, this assumption has been questioned (Thomas \& KarmiloffSmith, 2002). Rather than the atypical case representing the development of some parts of the typical system (here, the resilient properties), what we may see is a qualitatively atypical system that is not directly comparable to the typical case, principally due to compensatory changes.

Figure 1 represents this idea more concretely. Goldin-Meadow compares the use of hand signs in communication in homesign, in sign language, and in gesturing in typically developing (TD) children and adults. The difference (or subtraction) between homesign and sign language in the manual modality reveals resilient properties, while TD gesture represents a baseline of spontaneous gesture. Figure 1 depicts the idea that communication employs at least three sorts of information: that transmitted orally, that transmitted by hand gestures, and pre-existing shared knowledge / context between speaker and listener of what may be intended by the speaker in a communicative act. In the TD individual, most of the weight is placed in the oral channel, relatively little in gesture, and relatively little in shared context. In the deaf individuals, the oral channel is not available. Homesign and sign language represent two different 
compensatory re-weightings. Homesign relies heavily on shared context, as well as increasing the weighting of the hand channel. Sign language instead relies most heavily on the hand channel, relying less on shared context. The reduced reliance on shared context enables it to be acquired by communities of speakers who do not know each other well, but requires more information to be transmitted via the hand channel. Such re-weighting may not only be a characteristic of a developmental state but also a flexible strategy, such as when TD individuals increase their gesturing whilst attempting to communicate with someone who speaks a different language.

In this view, then, language development under atypical circumstances is about flexibly re-weighting the use of different information sources to achieve effective communication. Subtraction methodology applied to the single channel of hand movements would not serve to reveal the hidden resilient properties that covertly act during typical language acquisition. Rather, what is observed in homesign is a different strategy that forces extra information through a gesture communication channel while relying much more heavily on a shared understanding of context of what is probably intended. Such understanding is present in a family unit with an extensive shared history of experience.

\section{Can individual differences data really tell us about species universal developmental mechanisms?}

The second caution is how one moves towards a mechanistic understanding based on the type of data provided by Goldin-Meadow's synthesis, the explicit goal stated in the title of Goldin-Meadow's article. How can we anchor debates about notions such as innate 'principles', 'biases', 'structures', and 'ideas', and 
what it means for them to 'govern' development? How can we evaluate the proposal that 'individual differences ... provide insight into how children bring the [species universal] resilient properties to bear on language learning'? Computational modelling of development provides one tool to address these issues. It is instructive to see what is involved in building a model to simulate some of the empirical effects that Goldin-Meadow refers to, such as those related to how socio-economic status (SES) effects on language development interact with the effects of early brain damage.

There are four steps necessary. First, it is necessary to have a model that simulates development trajectories of language acquisition. This requires specification of the learning mechanisms that all individuals share, and specification of the language environment to which all individuals are exposed. Second, it is necessary to stipulate a theory of individual differences that comes in (at least) two parts: what varies between individuals in their learning mechanisms (let us call them intrinsic factors) and what varies in the language environments with which they interact (let us call them extrinsic factors). Third, to simulate a given set of data, it is necessary to make an assumption about the relative extents (or weighting) that intrinsic and extrinsic factors contribute to individual differences in that population. And fourth, it is necessary to simulate the development of large numbers of children, to observe the modulations that individual differences factors produce in developmental trajectories. These modulations may embody potentially complex interactions between intrinsic and extrinsic factors. What is involved, then, is modelling at a population level, so that individual differences can be properly considered within a developmental framework. 
One recent model provides an example. It sought to simulate SES effects on language development in the domain of inflectional morphology (Thomas, Forrester \& Ronald, 2013). The model captured data on English past tense acquisition, where children usually find regular verbs easier to learn than irregular verbs. For current purposes, we might consider this difference a dimension of difficulty in language acquisition. An artificial neural network was employed as the learning mechanism; intrinsic variations were produced by small differences in multiple parameters affecting network construction, activation dynamics, and plasticity; the effects of SES were simulated by a manipulation of the quantity of language information networks were exposed to, in line with the research that Goldin-Meadow reviews; and several populations of children were simulated where intrinsic and extrinsic factors made different relative contributions to producing individual differences.

Now, let us take a couple of qualitative empirical observations noted by Goldin-Meadow: children with reduced processing capacity due to early brain lesions show exaggerated difficulty on the more challenging parts of language (Stiles et al., 2014); and variations in SES cause greater divergence of developmental trajectories in syntax in children with reduced processing capacity compared to TD children (Rowe et al., 2009). Figure 2 shows developmental trajectories from the Thomas et al. model for two populations, each of 1000 simulated children. In one population, intrinsic factors were primarily responsible for producing individual differences (i.e., children had very different capacities to learn). In the other, intrinsic and extrinsic (SES) factors were equally responsible. Networks were separated into groups according to their learning capacity. Collapsing across SES groups, the plot shows that the 
impact of task difficulty (the difference between regular and irregular verb acquisition) was exaggerated in low learning capacity networks, per Stiles et al. (2014). Figure 3 now splits the population according to SES. Panels (a) and (b) show regular and irregular verb trajectories for the population with mainly intrinsically caused individual differences, (c) and (d) for equally intrinsically and extrinsically (SES) caused individual differences. Did low capacity exaggerate the divergent effects of SES on trajectories? Yes, in three out of four cases (b-d), most clearly for regular verbs in the equal intrinsic-extrinsic population. Low capacity networks were increasingly impacted across development by having poorer language input. Only when intrinsic sources of variation were a strong limiting factor did this not occur, in Fig. 3(a).

We discuss the model here for four reasons. First, it illustrates the kind of framework necessary to consider the relationship between individual differences data and species universal development with respect to mechanism. Second, it demonstrates that interactions of task difficulty, learning capacity, and SES such as those reviewed by Goldin-Meadow can emerge from relatively generic associative networks. Third, in the model, the advantage of regular verbs over irregular verbs held in all the simulated conditions presented - this 'resilient' property wasn't traceable to innate structures, it was a property of the task; it just happened to be invariant over the conditions considered. The source of resilient properties must therefore be interpreted with caution. Last, the model shows the potential trap in too readily drawing inferences about species universal mechanisms from individual differences data: Figure 3(a) indicates that variations in the language environment associated with SES had little impact on individual differences in developmental trajectories (where intrinsic factors 
were more important). One might conclude that the environment was not important for language development based on individual differences. But in this simulated population, species universal development was 100\% experiencedependent. Variations in development were mainly due to intrinsic factors.

\section{Variation in gesture and communication beyond humans}

Our third caution also concerns species universals. An important complementary approach is to allow these universals to vary in comparative studies, thereby providing a broader evolutionary context for communication. Recent data suggest that comparative studies examining gesture and communication in primates may offer a valuable insight into species universals in humans.

Due to their phylogenetic proximity to humans, great apes represent an excellent proxy to investigate universals of human communication. The ape model may reflect the ancestors of modern humans prior to the emergence of language but after the emergence of left hemisphere dominant regions for language processing (Cantalupo, Pilcher \& Hopkins, 2003; Spocter et al., 2010).

Our shared evolutionary history of left cerebral dominance for motor articulation of the hands and mouth may explain why, like humans, captive and wild chimpanzees express population-level right hand biases for the production of communicative gesture (e.g., Hopkins et al., 2005; Hobaiter \& Byrne 2013). Great apes are also frequently reported as right-handed tool users (e.g., Forrester, Quaresmini, Leavens, Mareschal \& Thomas, 2013; Tabiowo \& Forrester, 2013). Moreover, right-hand dominant object manipulation sequences have been likened to simple action syntax (e.g., Byrne \& Byrne 1991). Thus, language features such as simple syntactic structures and hierarchical 
organisation are likely to have origins in behaviours inherited from a last common ancestor of humans and apes. This position is supported by neurophysiological evidence: Broca's area has been argued to be a left hemisphere dominant supra-modal processor for routine, sequenced action that was later adopted for behaviours that require action syntax (e.g., tool use) and ultimately behaviours that require an internal syntax (e.g., language) (see, Tettamanti \& Weniger, 2006). Additional neurophysiological evidence demonstrates: (1) shared neural correlates for language and tool use in Broca's area (Higuchi et al., 2009) and (2) that language practised through either speech or gesture is controlled by the same motor regions (Gentilucci, Benuzzi, Gangitan \& Grimaldi, 2001). The exploration of the communicative predecessors to human language via great ape models has the potential to inform about the universal, phylogenetic properties of language and their neural correlates. Great ape models further provide an opportunity to investigate resilient properties of communication systems and their developmental trajectories by manipulating the social environment. For obvious ethical reasons, we do not manipulate children's exposure to social engagement for scientific purposes. However, investigations of non-human primate behaviour allow for the exploration of cognitive outcomes associated with a range of different rearing histories. For example, in typically developing children, pointing gestures generally coincide with the production of first words (e.g., Bates \& Snyder, 1987). The general consensus from the literature is that apes do not comprehend the pointing gestures of humans (e.g., Tomasello, 2006). However, recent metaanalyses have revealed that cross-fostered apes (exposed to artificial languages and/or natural sign languages), produce and comprehend pointing gestures, in 
addition to a host of other communication processes thought to be unique to humans (e.g., understanding the direction of attention in others) (Lyn, Russell \& Hopkins, 2010). Conversely, apes raised in communication-deprived environments (e.g., biomedical institutions, reared in single cages) do not develop these communicative capabilities. The 'Lived Experiences' model further demonstrates that an ape's ability to engage in joint attention (a foundational component of human communication) can be significantly influenced by the individual's past engagement experiences (Bard et al., 2014). These findings clearly demonstrate that even with the phylogenetic neural architecture intact, exposure to culturally relevant stimuli is required to trigger the development and acquisition of communication skills. There is no reason to believe that the case would be any different for language acquisition during human development. Lastly, investigations of ape communication development that allow for the manipulation of the age at which a communication-rich environment is introduced provide an additional powerful tool to offer further perspectives on the resilient features of human language.

Studies of human language generally neglect the fact that animals use all of their senses to send and receive information about the world (e.g., Darwin 1872). Investigations of child language development assume that intentional communication is restricted to hand and mouth articulation, even though it is widely accepted humans use a rich repertoire of verbal and nonverbal signals to communicate. Like humans, great ape communication is embodied and incorporates elements of posture, eye gaze, facial expression, direction of attention, limb action and proximity to the communication recipient (Forrester, 2008). Non-human primate and human communication is multimodal. Children 
reared in an environment devoid of language structure are likely to use embodied communication skills in a structured manner to communicate intent, beyond simply vocal or gestural methods. Perhaps we need to broaden our definitions of 'communication' and 'language' to take into account the complex multimodal nature of communicative behaviour. The investigation of modern human cognitive function requires a dual perspective, as the evolution and development of language functions are inextricably linked.

\section{Acknowledgements}

This work was supported by UK ESRC grant RES-062-23-2721.

\section{References}

Bard, K. A., Bakerman, R., Boysen, S. T., \& Leavens, D. A. (2014). Emotional engagements predict and enhance social cognition in young chimpanzees. Developmental Science, 17(5), 682-696. doi: 10.1111/desc.12145

Bates, E., \& Snyder, L. S. (1987). The cognitive hypothesis in language development. In E. Ina, C. Uzgiris, \& E. J. McVicker Hunt (Eds.), Infant performance and experience: New findings with the ordinal scales (pp. 168-204). Urbana, IL, USA: University of Illinois Press.

Byrne, R. W., \& Russon, A. E. (1998). Learning by imitation: a hierarchical approach. Behavioral and Brain Sciences, 2, 667-721.

Cantalupo, C., Pilcher, D. L., \& Hopkins, W. D. (2003). Are planum temporale and sylvian fissure asymmetries directly correlated? A MRI study in great apes. Neuropsychologia, 41, 1975-81. doi:10.1016/S0028-3932(02)00288-9. 
Darwin, C. (1872). The Expression of the Emotions in Man and Animals. 2nd edition. New York: D. Appleton. http://dx.doi.org/10.5962/bhl.title.4820.

Forrester, G. S. (2008). A multidimensional approach to investigations of behaviour: Revealing structure in animal communication signals. Animal Behaviour, 76, 1749-1760. doi:10.1016/j.anbehav.2013.09.010.

Forrester, G. S., Quaresmini, C., Leavens, D. A., Mareschal, D., \& Thomas, M. S. C. (2013). Human handedness: An inherited evolutionary trait. Behavioural Brain Research, 237, 200-6. doi: 10.1016/j.bbr.2012.09.037.

Gentilucci, M., Benuzzi, F., Gangitano, M., \& Grimaldi, S. (2001). Grasp with hand and mouth: A kinematic study on healthy subjects. Journal of Neurophysiology, 86(4), 1685-1699.

Goldin-Meadow, S. (2015). Studying the mechanisms of language learning by varying the learning environment and the learner. Language, Cognition and Neuroscience. doi: 10.1080/23273798.2015.1016978.

Higuchi, S., Chaminadeb, T., Imamizua, H. \& Kawatoa, M. (2009). Shared neural correlates for language and tool use in Broca's area. Neuroreport, 20, 13761381. doi: 10.1097/WNR.0b013e3283315570.

Hobaiter, C., \& Byrne, W. B. (2013). Laterality in the gestural communication of wild chimpanzees. Annals of the New York Academy of Sciences, 1288, 9-16. doi: $10.1111 /$ nyas. 12041

Hopkins, W. D., Russell, J., Freeman, H., Buehler, N., Reynolds, E. \& Schapiro, S. J. (2005). The Distribution and Development of Handedness for Manual Gestures in Captive Chimpanzees (Pan troglodytes). Psychological Science, 16(6), 487-493. doi: 10.1111/j.0956-7976.2005.01561.x 
Lyn, H., Russell, J. \& Hopkins, W. (2010). The impact of environment on the comprehension of declarative communication in apes. Psychological Science, 21, 360-365. doi: 10.1177/0956797610362218.

Rowe, M. L., Levine, S.C., Fisher, J., \& Goldin-Meadow, S. (2009). Does linguistic input play the same role in language learning for children with and without early brain injury? Developmental Psychology, 45, 90-102. doi: $10.1037 / \mathrm{a} 0012848$

Spocter, M. A., Hopkins, W. D., Garrison, A. R., Bauernfeind, A. L., Stimpson, C. D., Hof, P. R., et al. (2010). Wernicke's area homologue in chimpanzees (Pan troglodytes) and its relations to the appearance of modern human language. Proceedings of the Royal Society B, 277, 2165-74. doi: $10.1098 /$ rspb.2010.0011

Stiles, J., Reilly, J. S., Levine, S. C., Trauner, D., \& Nass, R. D. (2014). Neural plasticity and cognitive development: Insights from children with perinatal brain injury. Oxford University Press, in press.

Tabiowo, E. \& Forrester, G. S. (2013). Structured bimanual actions and hand transfers reveal population-level right-handedness in captive gorillas. Animal Behaviour, 68, 1049-1057. doi:10.1016/j.anbehav.2013.09.010.

Tettamanti, M. \& Weniger, D. (2006). Broca's area: a supramodal hierarchical processor? Cortex, 42, 491-494. doi:10.1016/S0010-9452(08)70384-8.

Thomas, M. S. C. \& Karmiloff-Smith, A. (2002). Are developmental disorders like cases of adult brain damage? Implications from connectionist modelling. Behavioral and Brain Sciences, 25(6), 727-788. doi: http://dx.doi.org/10.1017/S0140525X02000134 
Thomas, M. S. C, Forrester, N. A., \& Ronald, A. (2013). Modeling socio-economic status effects on language development. Developmental Psychology, 49(12), 2325-43. doi: $10.1037 / \mathrm{a} 0032301$

Tomasello, M. (2006). Why Don't Apes Point? In N. J. Enfield \& S. C. Levinson (eds.), Roots of Human Sociality: Culture, Cognition and Interaction. Oxford \& New York: Berg, pp. 506-524. 


\section{Figure captions}

Figure 1: A schematic of the (possible) relative use of oral communication, gesture communication, and shared context to achieve transmission of a message in typical development, homesign, and sign language. The thickness of each arrow represents the extent to which each source of information is utilised. The use of hand signs is not directly comparable across conditions because each case represents a re-weighting of channels.

Figure 2: Simulated data from a population-level model of past tense acquisition designed to capture interactions between the effects of socio-economic status (SES) and learning ability on developmental trajectories. Data are shown for two simulated populations, one in which individual differences (ID) are mainly caused by intrinsic variations in learning ability, one in which intrinsic and extrinsic (SES) variations are equally responsible for producing individual differences. Trajectories across three points in development (early, mid, late) are split by learning capacity, with around 250 simulated individuals per group. For both populations, the difficulty of acquiring irregular versus regular verbs is exaggerated by low learning capacity.

Figure 3: Simulated trajectories from the population modelling, split by difference socio-economic status levels. (a) Trajectories for the population in which intrinsic variations in learning ability are mainly responsible for individual differences, for (a) regular and (b) irregular verbs. Trajectories for the 
population in which intrinsic and extrinsic (SES) variations are equally responsible for producing individual differences, for (c) regular and (d) irregular verbs. (b) to (d) all show that SES differences cause divergent trajectories for low learning capacity networks. 


\section{Figures}

Figure 1

Typical development

Listener

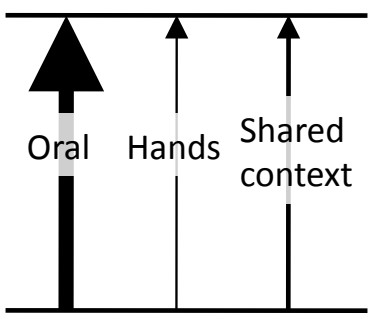

Speaker
Homesign

Listener

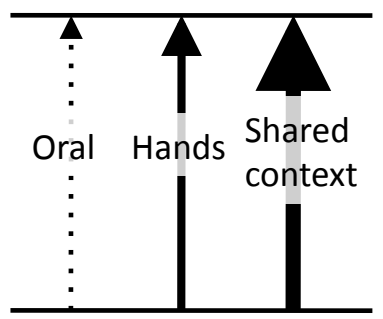

Speaker
Sign language

Listener

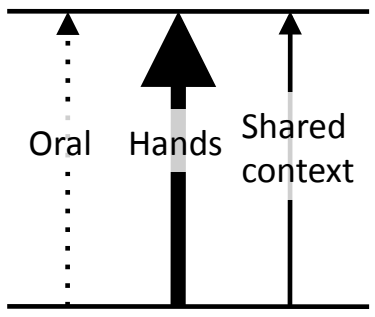

Speaker 
Figure 2

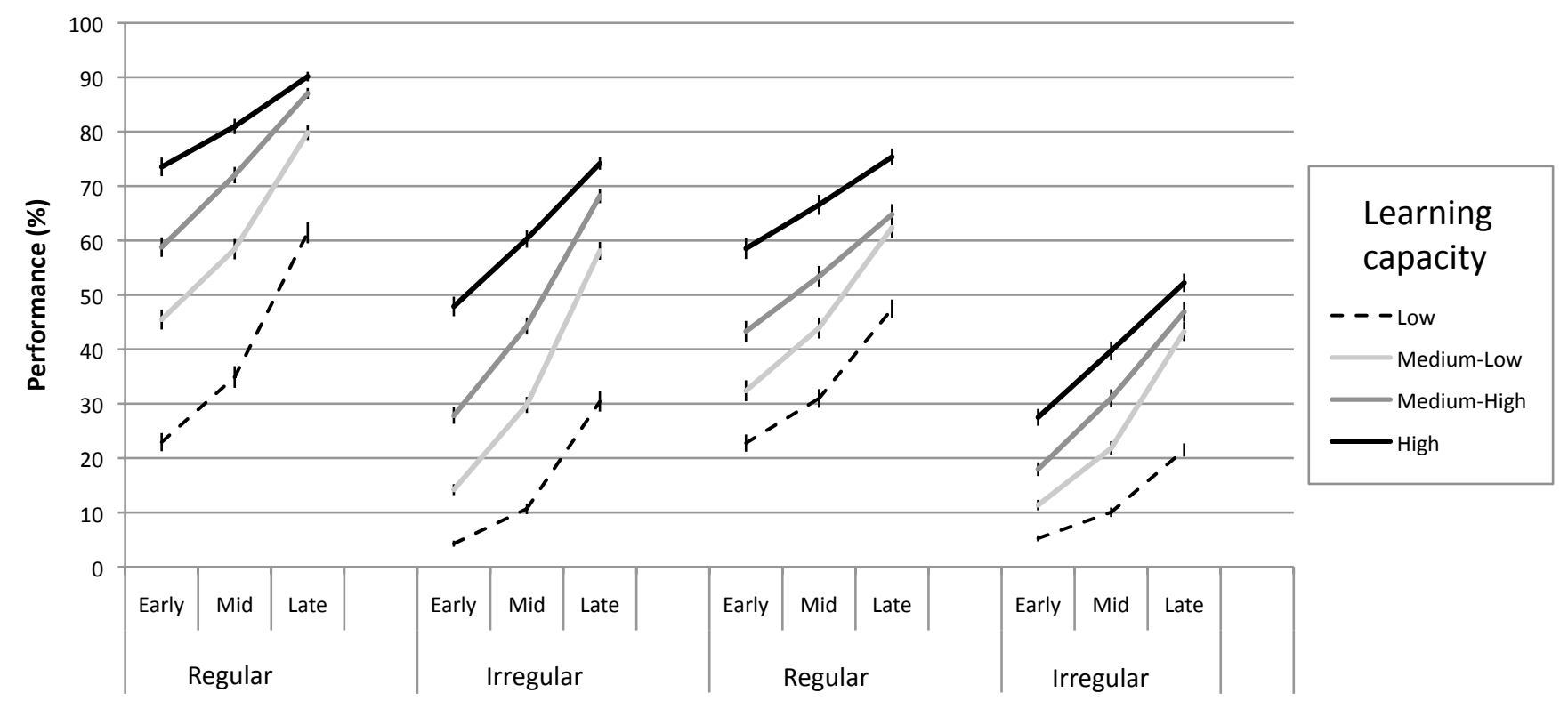

Population with ID caused mainly by intrinsic differences

Population with ID caused equally by intrinsic differences and SES 
Figure 3

(a)

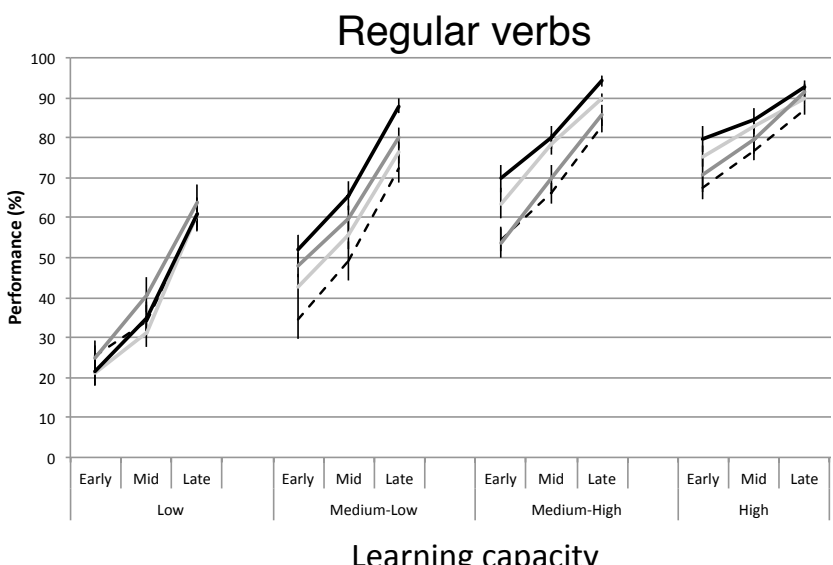

(c)

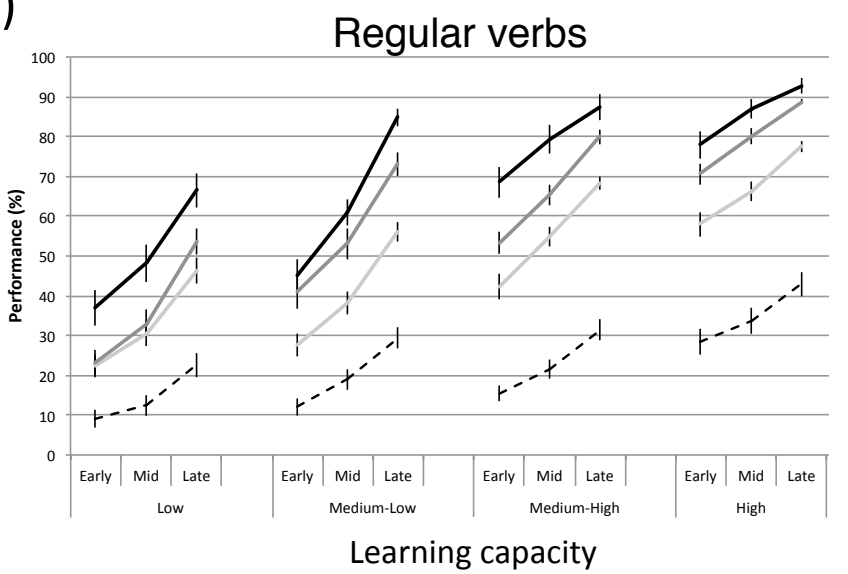

(d)

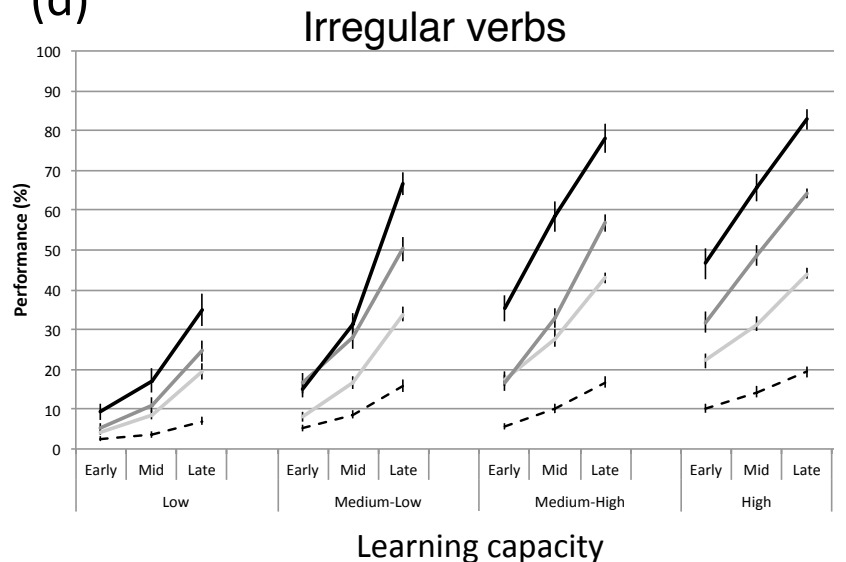

(b)

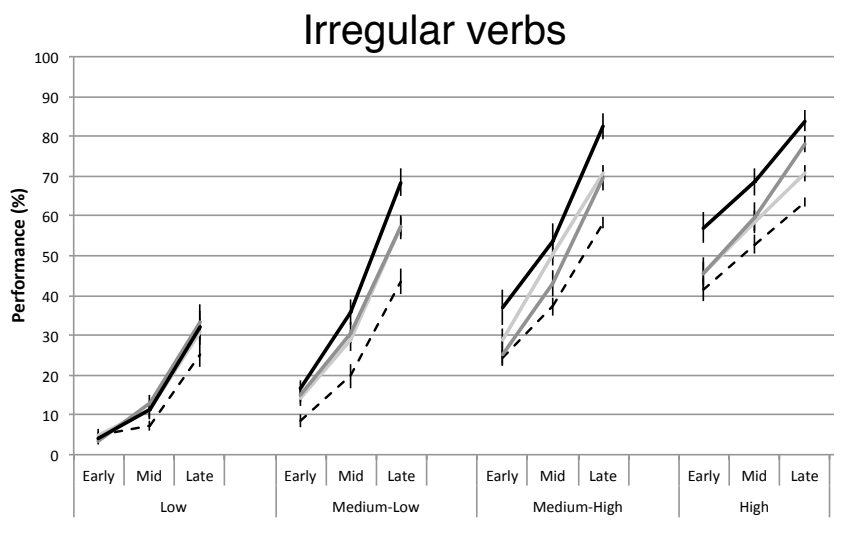

Learning capacity

capacity

SES:

Medium-Low
Medium-High

- High 